\title{
$\Rightarrow$ ATTENTION
}

\section{The focus of attention}

Selective attention helps the visual system to process the most behaviourally important information by preferentially routing such information across cortical networks. This selective routing is thought to rely on the synchronization of activity in different cortical neuron populations, but how this is achieved has been unclear. Now, Saalmann et al. show that the pulvinar, a higher-order thalamic nucleus, has a key role in this process.

In the ventral visual cortical pathway, information is processed in the $\mathrm{V} 4$ area and, subsequently, the temporo-occipital area (TEO), which are structurally connected to each other and to the pulvinar. Using diffusion tensor imaging and tractography, the authors showed in macaques that V4 and the TEO mainly connect to the ventral region of the pulvinar and have overlapping projection zones.

The authors placed electrodes in these three brain regions and recorded spike trains and local field potentials (LFPs) while the monkeys performed a visuospatial attention task. In this task, animals were presented with a spatial cue that drew attention to the position of a subsequently presented target. Monkeys attending the position of the cue, after the cue had disappeared, quickly recognized the target, which was surrounded by distracting stimuli, and depressed a lever to receive a reward.

Neuronal population activity in the pulvinar increased with cue or target presentation. Notably, target-linked activity was greater in animals that had attended the cue's position than in those that had not, indicating that attention to the cue's position increased target-induced pulvinar responses.

During the delay period between cue and target presentation, there was an increase in spike-field coherence between pulvinar neurons in monkeys attending the cue's position. This increase was most notable in the alpha-frequency band and reflected a rise in synchronized activity. A rise in such activity was also observed between V4 and the TEO with selective attention.

Strikingly, selective attention caused a rise in the coherence of LFPs in the alpha oscillatory frequency range, and hence an increase in synchronized activity, between the pulvinar and V4 and the pulvinar and the TEO, further supporting a role for the pulvinar in the monkey's attentional network.

Granger causality testing, which assesses the influence of one variable over another, revealed that in monkeys attending the cue's position during the delay period, pulvinar activity strongly influenced alpha oscillatory activity in $\mathrm{V} 4$ and the TEO, but $\mathrm{V} 4$ activity only weakly influenced TEO activity. Together, these findings suggest that pulvinar-cortical interactions are crucial for attention-induced synchronous activity in cortical regions.

Darran Yates

ORIGINAL RESEARCH PAPER Saalmann, Y. B. et al. The pulvinar regulates information transmission between cortical areas based on attention demands. Science 337, 753-756 (2012)

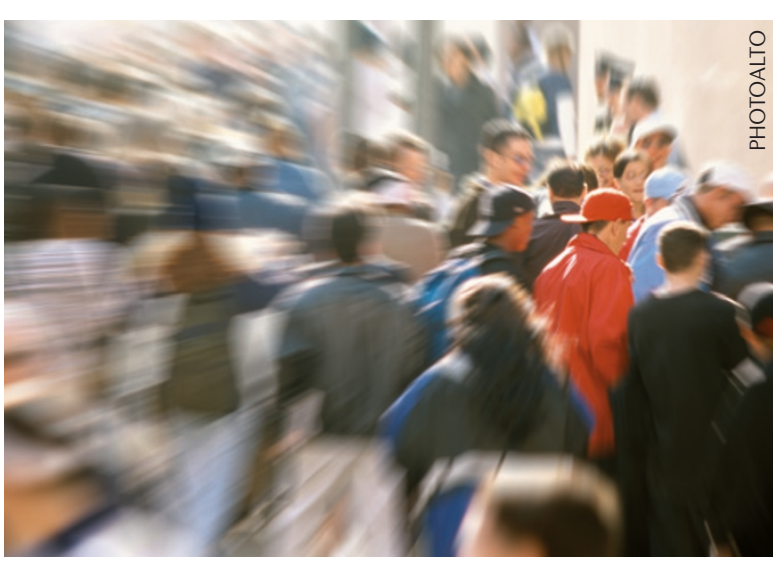

